\title{
Clinical and Psychosocial Characteristics of HIV-Positive and HIV-Negative Females with Opiate Dependence
}

\author{
Stan'ko EP' ${ }^{1}$, Igumnov $\mathbf{S A}^{2}$, Nestsiarovich $\mathbf{A}^{\mathbf{3}}$, Grinevich $\mathbf{E V}^{\mathbf{4}}$ \\ ${ }^{1}$ Grodno State Medical University, Grodno, Belarus \\ ${ }^{2}$ National Research Center for Addiction Problems, Moscow, Russia \\ ${ }^{3}$ Belarusian State Medical University, Minsk, Belarus \\ ${ }^{4}$ Belarusian State University, Minsk, Belarus
}

Article Details
Article Type: Research Article
Received date: $23^{\text {rd }}$ April, 2019
Accepted date: $28^{\text {th }}$ May, 2019
Published date: $29^{\text {th }}$ May, 2019

*Corresponding Author: Sergey Igumnov, National Research Center for Addiction Problems, Moscow, Russia. E-mail: sigumnov67@gmail.com

Citation: Stan'ko EP, Igumnov SA, Nestsiarovich A, Grinevich EV (2019) Clinical and Psychosocial Characteristics of HIVPositive and HIV-Negative Females with Opiate Dependence. J Pub Health Issue Pract 3: 140. https://doi.org/10.33790/ jphip1100140.

Copyright: (C2019, This is an open-access article distributed under the terms of the Creative Commons Attribution License 4.0, which permits unrestricted use, distribution, and reproduction in any medium, provided the original author and source are credited.

\begin{abstract}
The objective of the study was to explore the clinical-psychological and social characteristics of injecting drugs women users (IDWU) with opiate dependence with different HIV status living on the territory of the Republic of Belarus based on multi-dimensional assessment of opiate dependence dynamics in order to develop therapeutic measures for relapse prevention and achievement of the long-term remission.

Materials and methods: Totally 217 IDWU were examined. The research cohort was randomized into 2 groups: HIV-positive women (HPW) (104 persons, mean age 30.8 years $(\mathrm{SD}=4.86)$ and HIV-negative women (HNW) (113 persons, mean age 29.9 years ( $\mathrm{SD}=6.67)$. The level of patients social functioning, quality of life, craving for narcotic substances were studied by means of the Scale of the social and functioning, SF-36 and the Scale of the craving for drugs. The particularities of the social functioning and life quality of women with opiate addiction and different HIV status were described.

The results: show that the majority of IDWU were characterized by frequent work replacement and labor skipping (27/25.9\% in HPW group vs $30 / 26.5 \%$ in HNW group; $\mathrm{P}>0.05$ ). $21 / 20.1 \%$ HPW vs $15 / 13.2 \% \mathrm{HNW}(\mathrm{P}<0.05)$ was characterized by low qualification, $43 / 43.3 \%$ HPW vs $41 / 36.2 \%$ HNW ( $P>0.05)$ - vocational education. HPW have more severely impaired psychosocial parameters compared to HNW. Family climate can be described as hostile aggressive (84/80.7\% in HNW group vs $69 / 61.0 \%$ in HNW group; $\mathrm{P}<0.05$ ). For women of both groups were characteristic parental alcohol abuse (18/17.3\% in HPW vs $16 / 14.1 \%$ in HNW; $\mathrm{P}>0.05)$, and high level of parental divorces $(24 / 23.1 \%$ in HPW vs $32 / 28.3 \%$ in HNW; $\mathrm{P}>0.05$ ). It was found that the treatment itself allows to diminish the problems associated with drug use and HIV infection. Nevertheless, the short-term treatment course can only provide the clinical improvement but not stabilization of psychosomatic state of HIV-positive patients with opiate dependence.
\end{abstract}

Conclusion: The results of the study indicates the vast majority of women who are injecting drug users, living in Belarus, can be characterized by high level of social functioning disturbances including domestic, social and professional relationship. They need for development of specific tactics of management to suit their needs, including the long-term complex treatment and rehabilitation programs.

Key words: women, quality of life, social functioning, complex treatment and rehabilitation programs, opiate dependence, HIVstatus.

Relevance of the problem of gender differences among drug users

According to the data presented in the world drug report 2016 of the United Nations Office on drugs and crime, there are significant gender differences among drug users. Thus, men are three times more likely to use cannabis, cocaine and amphetamines than women, and women are more likely to use non-medical tranquilizers and opioids [1]. Women are more likely to use prescription benzodiazepines and narcotic analgesics for non-medical purposes $[2 ; 3]$. The use of prescription drugs in women increases with age and reaches a peak by $30-40$ years. Women use drugs more often in order to overcome stressful situations in their lives; they are prescribed drugs and sedatives more often than men $[4 ; 5]$.

According to H. A. Whiteford et al. [5], one third of IDUs worldwide are women and girls; the number of injecting drug women users (IDWUs) is approximately $0.11 \%$ of the world's female population.

Risk factors for antisocial behavior and drug abuse among women are sexual coercion in childhood and intimate partner violence. Violence affects women's mental, physical and reproductive health. $20 \%$ of women who have experienced violence in the past develop a mental disorder, most often depression or post-traumatic stress disorder [6]. Consumption of surfactants in women more often than in men is accompanied by the development of various mental disorders. Thus, in the EU, comorbid depression develops much more often in women than in men. The prevalence of depression in this group of women is twice that of women in general [7]. 
In general, women start using drugs later than men. However, in the course of drug abuse the level of consumption of cannabis, opioids and cocaine among women growing faster than among men, before developing disorders on the basis of receiving surfactant, compared with men, women who use heroin, at least use it with the help of injections in smaller doses and for a short time, are younger, and are often influenced by drug-using sexual partners, who often do the first injection of drugs to a woman [6].

The transition to IPRs among women may be due to personal characteristics or external circumstances. This may be a consequence of problems in personal life, affective and anxiety disorders, desire to lose weight, struggle with exhaustion, anesthesia, self-treatment of mental disorders, physical or sexual violence experienced in childhood, involvement in the sex industry and communication with injecting drug users (IDUs) [6].

Many more often than not, women who practice pit report using needles in combination, explain that they are not aware of the risks involved, are unable to buy syringes/needles from pharmacies, or fear of over-policing, using shared needles as a sign of love or trust in a partner. "Dirty" injections can cause damage to the veins and cause serious complications. IDWU are experiencing problems such as fatigue, loss of weight, withdrawal symptoms of pain, depression and a suicide attempt, many of them suffer from sexually transmitted diseases, viral hepatitis. For these women, access to health care is mainly hampered by the fact that society condemns women who inject drugs more than men [6].

Women heroin users, younger age compared with men who rarely consume it by injection in smaller doses and for a shorter time, often under the influence of sexual partners, using drugs that often make the woman the first injection of drugs. Often the transition to injecting drug use (IDU) among women is due to personal characteristics of women or external circumstances due to problems in their personal lives, affective or anxiety disorders, desire to lose weight, fight against exhaustion, anesthesia, self-treatment of mental disorders, physical and sexual violence experienced in childhood, involvement in the sex industry or communication with IDUs [1].

Many women who practice pit report sharing needles, either because they are not aware of the risks, or because they are unable to buy needles from pharmacies, or because they fear the police, or because they share needles as a sign of love or trust in their partner. Lack of IDU experience can lead to vein damage and cause serious complications. IDWU often experience fatigue, weight loss, pains, depression, suicide attempt, many of them suffer from sexually transmitted diseases, hepatitis. Access to health care for them is difficult because of the public condemnation [1].

For the period between 1999 and 2010, mortality among IDWU, in particular the use of opioid analgesics prescription in the USA increased five-fold among men by 3.6 times. More than 15,000 women died from a drug overdose in the United States in 2012. From 2007 to 2008 in England and Northern Ireland, overdose mortality among women increased by $17 \%$, among men by $8 \%$ [3].

The purpose of this study is to study the medical and social characteristics of HIV-positive (HPW) and HIV-negative (HNW) women who inject drugs (opioid group) (IDWU).

\section{Materials and methods of research}

The study design is defined as an analytical combined (crosssectional and longitudinal) study with regular monitoring (6-fold) of the monitored parameters before and after treatment, at the stage of follow-up-after 1,3,6 and 12 months from the beginning of follow-up.

This study was conducted on the basis of Grodno State Medical University on the instructions of the research program "To develop criteria for clinical and social functioning, to assess the quality of life and maladaptation of injecting drug users (IDUs) suffering from various stages of HIV infection" (№ State Registration 201501548), with the scientific and methodological support of the National Research Center for Addiction Problems - a branch of the National Medical Research Center of Psychiatry and Narcology named after V. P. Serbsky" Ministry of Health of Russia.

The clinical study was performed in accordance with GCP rules, according to the Protocol using an individual registration data card. To conduct the study was developed by an individual registration form that includes the questions that health and social aspects of IDWU. 217 female patients with dependence on opioids living in the Republic of Belarus and undergoing stationary treatment in health care institutions of psychiatric profile were examined. The examined persons were randomized into 2 groups: group $1-104$ HIV-positive (HPW) and HIV-negative (HNW) women of middle age $30.8(\mathrm{SD}=4.86)$ years and group $2-113 \mathrm{HNW}$ of middle age $29.9(\mathrm{SD}=6.67)$ years. The average age of HIV infection in the HPW group was within Me 24.0 (21.0-27.0).

\section{Results and discussion}

The study showed a characteristic feature among the majority of surveyed IDWU such as the lack of a stable official position. On a temporary or seasonal job were $18 / 17.3 \%$ of HPW and 20/17.6\% $\mathrm{HNW}$; frequent changes of places of work, interruptions were observed in $27 / 25.9 \%$ of HPW and 30/26.5\% HNW; not working, not studying, shied away from work $63 / 60.5 \% \mathrm{HPW}$ and $53 / 46.9 \% \mathrm{HNW}$; were employed in low-skilled jobs $24 / 23.1 \%$ of HPW and $19 / 16.8 \%$ of HNW. Systematic violations of labor discipline in 16/15.3\% HPW and $9 / 7.9 \% \mathrm{HNW}$ were shown in the form of frequent layoffs; in 15/14.4\% HPW and 9/7.9\% HNW - administrative penalties; in 19/18.2\% HPW and 16/14.1\% HNW - conflict relations /negative attitude to work. Disability had $3 / 2.88 \%$ of HPW and $1 / 0.88 \%$ of HNW.

Without a profession 45/43,2\% HPW and 38/33,6\% HNW, workers $55 / 52,8 \%$ HPW and 52/46,0\% HNW, employees $-6 / 5,7 \%$ HPW and 22/19,4\% HNW. Low educational level (incomplete secondary education) was typical for $21 / 20,1 \%$ HPW and $15 / 13,2 \%$ HNW, secondary education - 43/41,3\% HPW and 41/36,2\% HNW, secondary special education $-36 / 34,6 \%$ HPW and $43 / 38,0 \%$ HNW.

Their own families had $49 / 47.1 \%$ of HPW and $48 / 42.4 \%$ of HNW. Were in primary marriage $31 / 29.8 \% \mathrm{HPW}$ and $37 / 32.7 \% \mathrm{HNW}$, repeated $-13 / 12.5 \%$ HPW and 14/12/3\% HNW, civil - 18/17.3\% HPW and 19/16.8\% HNW. Divorced were 20/19.2\% HPW and 24/21.2\% HNW. Had children 72/69.2\% HPW and 73/64.6\% HNW. Lived with own family $31 / 29.8 \% \mathrm{HPW}$ and $37 / 32.7 \% \mathrm{HNW}$. Most of the time was spent among unfamiliar people, came home only for the night $21 / 20.1 \%$ of HPW and $9 / 7.6 \%$ of HNW $(p<0.05)$.

The situation in the family was characterized by the dominance of conflict relations in $84 / 80.7 \%$ of HPW and $69 / 61.0 \%$ of HNW, alcohol abuse of parents or one of them $-18 / 17.3 \%$ of HPV and $16 / 14.1 \%$ of HNV, lack of sufficient means of livelihood, low income in the family $-57 / 54.8 \%$ of HPW and $45 / 39.8 \%$; of $\mathrm{HNW}(\mathrm{p}<0.05)$, divorce of parents $-24 / 23.1 \%$ of HPW and $32 / 28.3 \%$ of HNW.

Age of the first drug trial was $18.1(\mathrm{SD}=3.4)$ among HPV and 20.4 (SD=4.8) among HNV. Opiates and opioids were the main drug used in $77 / 74.0 \%$ of HPW and $93 / 82.3 \%$ of HNW. Cannabinoids were used by $10 / 9.6 \% \mathrm{HPW}$ and $8 / 7.0 \%$ HNW, psychostimulants - 7/6.7\% HPW and 9/7.9\% HNW, tranquilizers - 16/15.3\% HPW and $1 / 0.8 \% \mathrm{HNW}(\mathrm{p}<0.001)$, barbiturates $-4 / 3.8 \% \mathrm{VPN}$ and $2 / 1.7 \%$ VNN $(\mathrm{p}<0.05)$. The main way the drug is taken at $84 / 80.7 \%$ of HPW and 103/91.1\% HNW was only injecting. The combination of different methods of administration of drugs was mentioned by $9 / 8.6 \%$ of HPN and $1 / 0.8 \%$ of HNW ( $<<0.01)$. At the same time, non-disposable syringes/needles were used by $46 / 44.2 \%$ of HPW and $11 / 9.7 \%$ of HNW $(\mathrm{p}<0.001)$, ignoring the sterility of the drug or the container in which it was $68 / 65.3 \%$ of HPW and $53 / 46.9 \%$ of HNW $(\mathrm{p}<0.05)$. 
The leading motive of drug abuse in $21 / 20.1 \%$ of HPW and $31 / 27.4 \%$ of HNW was the search for new pleasant sensations and curiosity. The desire to facilitate contact with people, to achieve the realization of sexual desire experienced $10 / 9.6 \%$ of HPW and 9/7.9\% HNW, to show his independence - 22/21.1\% HPW and 35/30.9\% HNW, induce euphoria, a pleasant feeling of altered mood $-41 / 39.4 \%$ of HPW and $49 / 43.3 \%$ (HNW), the desire to neutralize the negative emotional experiences $-24 / 23.1 \%$ of HPW and $27 / 23.8 \%$ HNW, to get out of the apathetic state, to "improve working efficiency" - 26/25.0\% HPW and 33/29.2\% HNW, the desire to get rid of painful manifestations of withdrawal state $-86 / 82.6 \%$ of HPW and $68 / 60.1 \% \mathrm{HNW}(\mathrm{p}<0.05)$; to take the drug in spite of yourself or others, to protest $-5 / 4.8 \% \mathrm{HPW}$ and $5 / 4.4 \% \mathrm{HNW}$.

Preferred to use the drug alone $17 / 16.3 \%$ HPW and $11 / 9.7 \%$ HNW $(\mathrm{p}<0.05)$, in the criminalized company $-83 / 79.8 \%$ HPW and $89 / 78.7 \%$ HNW. If it is impossible to get the "main" drug, used other psychoactive substances (PAS) 41/39.4\% HPW and 41/36.2\% HNW, in combination with alcohol or drugs used drugs $44 / 42.3 \%$ HPW and $39 / 34.5 \% \mathrm{HNW}$, a few PAS (surfactants) with the presence of the preferred drug $-3 / 2.8 \% \mathrm{HPW}$ and $11 / 9.7 \% \mathrm{HNW}(\mathrm{p}<0.05)$.

The duration of drug use without dependence from $31 / 29.8 \%$ of HPW and $28 / 24.7 \%$ of HNW up to 1 month up to 2 months $-11 / 10.5 \%$ of HPW and $16 / 14.1 \% \mathrm{HNW}, 3$ months $-1 / 0.9 \%$ of HPW and $5 / 4.4 \%$ HNW ( $<<0.05$ ), 6 months $-41 / 39.4 \%$ of HPW and $40 / 35.3 \%$ HNW, more than 6 months $-13 / 12.5 \%$ of HPW and $21 / 18.5 \%$ HNW. The emergence of signs of a withdrawal state from the beginning of the drug up to 1 month recorded at $35 / 33.6 \%$ of HPW and $25 / 22.1 \%$ HNW, up to $2-3$ months $-17 / 16.3 \%$ of HPW and $22 / 19.4 \% \mathrm{HNW}$, up to 6 months $-41 / 39.4 \%$ of HPW and $40 / 35.3 \% \mathrm{HNW}$, more than 6 months $-16 / 15.3 \%$ of HPW and $24 / 21.2 \%$ HNW. Illegal drug use in $62 / 59.6 \%$ of HPW and $34 / 30.0 \%$ HNW accounted for more than 10 years; $5-10$ years $-30 / 28.8 \%$ of HPW and $38 / 33.6 \%$ HNW, less than 5 year $-7 / 6.7 \%$ of HPW and $22 / 19.4 \%$ of HNW $(p<0.01)$.

The main motive for the break in drug use in $32 / 30.7 \%$ of HPW and $26 / 23.0 \%$ of HNW was the desire to reduce the dose of the drug; in $38 / 36.5 \%$ of HPW and $57 / 50.4 \%$ of $\mathrm{HNW}(\mathrm{p}<0.05)$ - the desire to completely abandon drug use, in $62 / 59.6 \%$ of HPW and $61 / 53.9 \%$ of HNW - financial difficulties associated with the acquisition of the drug, $42 / 40.3 \%$ of HPW and $37 / 32.7 \%$ of HNW - forced abstinence, in $49 / 47.1 \%$ of HPW and $37 / 32.7 \% \mathrm{HNW}$ - no permanent source of drug, $26 / 25.0 \%$ of HPW and $40 / 35.3 \%$ of HNW - moral pressure of relatives.

Attraction to drug use at 33/31.7\% HPW and 60/53.1\% HNW was situational, when released into the environment, $16 / 15.3 \%$ HPW - situational due to the "double stigma - HIV-infected female drug addicts", $42 / 40.3 \%$ of HPW and 55/48.6\% HNW - obsessive, escalating in situations of conflict, 68/65.3\% HPW and 42/37.1\% HNW $(p<0.05)$ - compulsive directional search of the drug or its substitutes, desire for contacts with the IDUs to obtain the illegal drug, 16/15.3\% of HPW and 16/14.1\% HNW - compulsive with a pronounced behavioral component with internal tension, excitability and increased irritability.

More than 10 cases of withdrawal states were noted: $37 / 35.5 \% \mathrm{HPW}$ and $23 / 20.3 \% \mathrm{HNW}$, up to $10-$ in $30 / 28.8 \% \mathrm{HPW}$ and $34 / 30.0 \%$ HNW, up to $5-$ in $25 / 24.0 \%$ HPW and $33 / 29.2 \%$ HNW, once in $8 / 7.6 \% \mathrm{HPW}$ and $10 / 8.8 \% \mathrm{HNW}$. The duration of withdrawal status in $5 / 4.8 \%$ HPW was more than 14 days, 14 days $-15 / 14.4 \%$ HPW and $1 / 0.8 \%$ HNW $(\mathrm{p}<0.01), 10$ days $-36 / 34.6 \%$ HPW and $27 / 23.8 \%$ HNW $(\mathrm{p}<0.05), 7$ days $-40 / 38.4 \%$ HPW and $62 / 54.8 \%$ HNW, 3-5 days $-3 / 2.8 \%$ HPW and $6 / 5.3 \%$ HNW. The severity of withdrawal state was mild in $3 / 2.8 \%$ of HPW and $10 / 8.8 \%$ of HNW $(\mathrm{p}<0.05) ; 79 / 75.9 \%$ of HPW and $77 / 68.1 \%$ of $\mathrm{HNW}$ - moderate and in $18 / 17.3 \%$ of HPW and $13 / 11.5 \%$ of HNW - severe. The main content of a withdrawal state in $82 / 78.8 \%$ of HPW and $76 / 67.2 \%$
HNW were somatic and neuro-vegetative disorders, tremor, sweating, disorders of the cardiovascular system; in $7 / 6.7 \%$ of HPW and $22 / 19.4 \%$ HNW $(p<0.05)$ - psychopathological disorders (fear, anxiety, depression, agitation); in $22 / 21.1 \%$ of HPW and $29 / 25.6 \%$ HNW - pain, convulsive disorders, $22 / 21,1 \%$ of HPW and $17 / 15.0 \%$ HNW - asthenic syndrome (sleep disturbances, general weakness, malaise).

The predominant symptoms in $70 / 67.3 \%$ of HPW and $78 / 69.0 \%$ of HNW in post-abstinent state were asthenic with general weakness, malaise, nausea, dizziness; in 28/26.9\% of HPW and 20/17.6\% of HNW were dominated by behavioral disorders; in $21 / 20.1 \%$ of HPW and $15 / 13.2 \%$ of HNW - aspontaneity. The duration of post-abstinent state was in $15 / 14.4 \%$ HPW and $5 / 4.4 \%$ HNW more than 1 month; in $67 / 64.4 \% \mathrm{HPW}$ and $62 / 54.8 \% \mathrm{HNW}$ - up to 1 month; in $17 / 16.3 \%$ HPW and $13 / 11.5 \% \mathrm{HNW}-14$ days; in $4 / 3.8 \% \mathrm{HPW}$ and $20 / 17.6 \%$ HNW - 3-7 days $(\mathrm{p}<0.01)$.

The main type of intoxication when using the drug in $65 / 62.5 \%$ of HPW and $61 / 53.9 \%$ of HNW was "pleasant relaxation", feelings of peace, deep self-contemplation; in 34/32.6\% of HPW and 48/42.4\% of HNW - desire for activity, feeling of fullness of life; in 50/48.0\% of HPW and 54/47.7\% of HNW - euphoria, emotional excitement, elation. However, it was observed modified forms of drug intoxication in the form of the predominance of low mood, sadness at $18 / 17.3 \%$ of HPW; dysphoria and irritability $-4 / 3.8 \%$ of HPW; aggressiveness on the background of emotional arousal - in $5 / 4.8 \%$ HPW vs $1 / 0.8 \%$ HNW ( $<<0.05$ ); demonstrative suicidal tendencies - in $2 / 1.9 \%$ of HPW; losing the sense of tact and shame, tearfulness - in $5 / 4.8 \%$ of HPW vs $1 / 0.8 \%$ of HNW $(\mathrm{p}<0.05)$.

Total abstinence from drug use was observed in $14 / 13.5 \%$ of HPW and $8 / 7.1 \%$ of HNW, partial - in $58 / 55.8 \%$ of HPW and $45 / 39.8 \%$ of HNW ( $<<0.05)$, spontaneous - in $8 / 7.7 \%$ of HPW and $3 / 2.7 \%$ of HNW ( $<<0.05)$; therapeutic remission - in $20 / 19.2 \%$ of HPW vs $26 / 23.0 \%$ of HNW. Early relapse (within 1 month after treatment) was observed in $40 / 38.5 \% \mathrm{HPW}$ and $35 / 31.0 \% \mathrm{HNW}$, up to 3 months - in $18 / 17.3 \% \mathrm{HPW}$ vs $13 / 11.5 \% \mathrm{HNW}$; 6 months $-7 / 6.7 \% \mathrm{HPW}$ vs $21 / 18.6 \%$ HNW ( $<<0.05$ ); up to 12 months- $24 / 23.1 \%$ HPW vs $17 / 15.0 \% \mathrm{HNW}$; more than 12 months $-12 / 11.5 \% \mathrm{HPW}$ vs $17 / 15.0 \%$ HNW.

The predominant symptoms in $70 / 67.3 \%$ of HPW and $78 / 69.0 \%$ of HNW in post-abstinent state were asthenic with general weakness, malaise, nausea, dizziness; in 28/26.9\% of HPW and $20 / 17.6 \%$ of HNW were dominated by behavioral disorders; in $21 / 20.1 \%$ of HPW and $15 / 13.2 \%$ of HNW - aspontaneity. The duration of post-abstinent state was in $15 / 14.4 \%$ HPW and $5 / 4.4 \%$ HNW more than 1 month; in $67 / 64.4 \% \mathrm{HPW}$ and $62 / 54.8 \% \mathrm{HNW}$ - up to 1 month; in $17 / 16.3 \%$ HPW and $13 / 11.5 \% \mathrm{HNW}-14$ days; in $4 / 3.8 \% \mathrm{HPW}$ and $20 / 17.6 \%$ HNW - 3-7 days $(\mathrm{p}<0.01)$.

Ability to critically assess the condition, partial criticism, preservation of drug addiction, emotional and volitional disorders, distortion of basic personal qualities were characteristic of 74/71.1\% HPW and $84 / 74.3 \% \mathrm{HNW}$; formal superficial attitude to drug intake, the desire to abandon anesthesia, the safety of critical prognostic abilities-7/6.7\% HPW and 22/19.4\% HNW ( $<<0.01)$; understanding of the consequences of intoxication, critical assessment of their condition with the desire to continue taking drugs giving thus "philosophical" justification - 8/7.6\% HPW; the desire to take the drug with full awareness of the disease, confidence in the ability to cope with the disease $-7 / 6.7 \%$ of HPW; did not hide the drug, was not treated, did not perform doctor's appointments $3 / 2.8 \%$ of HPW.

Experience of rehabilitation programs in $82 / 78.8 \%$ of HPW and $90 / 79.6 \%$ of HNW was absent. At the addictology stationary units of health institutions rehabilitated $4 / 3.8 \%$ of HPW and $14 / 12.3 \% \mathrm{HNW}$; in the center created by non-governmental organizations $-4 / 3.8 \%$ of HPW and $1 / 0.8 \%$ of $\mathrm{HNW}(\mathrm{p}<0.05)$; in the center, created by the 
Christian missions $-4 / 3.8 \%$ of HPW and $5 / 4.4 \% \mathrm{HNW}$ : commercial rehabilitation $-10 / 9.6 \%$ of HPW and $1 / 0.8 \%$ of HNW $(p<0.01)$.

Clinical manifestations of HIV infection were characterized in $57 / 54.8 \%$ of HPW during the month by a decrease in body weight for no apparent reason, in $37 / 35.6 \%$ of HPW - persistent causeless fever, in $15 / 14.4 \%$ - diarrhea, in $71 / 68.3 \%$ - an increase in lymph nodes, in $44 / 42.3 \%$ - unexplained constant night sweating, in $24 / 23.1 \%$ rapid fatigue, leading patients to spend most of the time lying down. $61 / 58.7 \%$ of HPW were receiving antiretroviral therapy.

Comorbid pathology was diverse. Organic mental disorder was revealed in $5 / 4.8 \%$ of HPW, affective disorders - in $34 / 32.7 \%$ of HPW, neurotic disorders - in 21/20.2\% of HPW. Diseases of the respiratory system were characteristic for $40 / 38.5 \%$ HPW, cardiovascular system disorders - for $19 / 18.3 \%$ of HPW; gastrointestinal tract pathology - for $28 / 26.9 \%$ of HPW; hepatopathy- for $98 / 94.2 \%$ of HPW; renal pathology - for $9 / 8.7 \%$ of HPW; skin diseases - for 9/8.7\% of HPW; malignant neoplasms - for $3 / 2.9 \%$ of HPW; central nervous system diseases - for $2 / 1.9 \%$ of HPW.

The changes of the way of life typical for HPW were diverse, among which $34 / 32,7 \%$ of HPW indicated incomplete education, $41 / 39,4 \%$ - change in habitual lifestyle, social activity, 83/79,8\% HPW - communication with persons with antisocial behavior, $59 / 56,7 \% \mathrm{HPW}$ - lack of freedom of choice, change of lifestyle, loss of their place in society, $44 / 42,3 \% \mathrm{HPW}$ - forced social isolation, lack of the usual circle of communication, friendly relations, 56/53,8\% HPW - problems in family relations, inability to start a family, have children, 64/61,5\% HPW - increased risk of job loss, employment, frequent change of employment, $80 / 76,9 \%$ of HPW - housing and financial problems, debts, $26 / 25 \%$ of HPW - lack of housing or registration at the place of residence, semi - legal existence, $35 / 33,7 \%$ of HPW - unhygienic lifestyle, malnutrition, $21 / 20,2 \%$ of HPW - loss of documents, difficulty of access to adequate health care, $25 / 24 \%$ of HPW -active continuation, due to despair, drug use and other 20/19, 2\% HPW - the need to take antiretroviral drugs at certain hours, not allowing passes, $7 / 6,7 \% \mathrm{HPW}$ - the need for food restrictions, $43 / 41,3 \% \mathrm{HPW}$ - a decrease in physical activity, 19/18,3\% HPW - psycho-physical fatigue from the forced use of antiretroviral drugs, $17 / 16,3 \% \mathrm{HPW}$ - the emergence of side effects when performing highly active antiretroviral therapy, $3 / 2,9 \%$ HPW - a sense of "peculiar medicinal smell" from your body, aversion to him, $6 / 5,8 \%$, VPN held ineffective antiviral therapy, $4 / 3,8 \%$ of HPW the appearance of visible defects in appearance, $2 / 1,9 \%$ of HPW knowing the terminal stage, the struggle with pain, asthenia.

$37 / 35.6 \%$ of HPW have the ability to perform their daily home, social and professional functions. 58/55.8\% of HPW experienced difficulties in performing household, social and professional duties could not perform home, social and professional functions 10/9.6\% of HPW.

\section{Summary}

The results of the study show that the majority of women who inject drugs of the opioid group are characterized by a high level of social functioning disorders, including domestic, social and professional responsibilities, while in IDWU with HIV-positive status these problems are more pronounced. This group of patients needs to develop special management tactics that take into account their medical and social characteristics and needs, including the development of long-term comprehensive treatment and rehabilitation programs.

\section{References}

1. Office of the United Nations Office on drugs and crime, world drug report 2016.

2. Simoni WL, Ritterand G, Strickler G. (2004) Gender and other factors associated with the nonmedical use of abusable prescription drugs, Substance Use and Misuse 39: 1-23.

J Pub Health Issue Pract

Volume 3. 2019. 140
3. ESPAD Report: (2007) Substance Use Among Students in 35 European Countries (Stockholm, Swedish Council for Information on Alcohol and Other Drugs, 2009).

4. UNODC, The Non-Medical Use of Prescription Drugs: Policy Direction Issues. - Vienna, 2011.

5. Global burden of disease attributable to mental and substance use disorders: (2013) findings from the Global Burden of Disease Study 2010/ H.A. Whiteford [et al.] // The Lancet 382: 1575-1586.

6. Report of the International narcotics control Board in 2016. United Nations: international narcotics control Board, new York, 2017.

7. EMCDDA, Comorbidity of Substance Use and Mental Disorders in Europe (Luxembourg, Publications Office of the European Union, 2015).

8. Occupational and demographic factors associated with drug use among female sex workers at the China Myanmar border $/ \mathrm{K}$. Hail-Jares [et al.] // Drug and Alcohol Dependence. - 2016. Vol. 161.

9. People who inject drugs in prison: HIV prevalence, transmission and prevention (2015) / K. Dolan [et al.] // International Journal of Drug Policy 26: S12-S15.

10. Worldwide prevalence and trends in unintentional drug overdose: (2015) a systematic review of the literature / S.S. Martins [et al.] // Am. J. Public Health 105: 29-49. 